\title{
ON REFLECTIONS AND ROTATIONS IN MINKOWSKI 3-SPACE
}

\author{
MELEK ERDOĞDU AND MUSTAFA ÖZDEMIR
}

Communicated by Abraham A. Ungar

Abstract. In this paper, we investigate the reflections in Minkowski three-space by three different approaches. Firstly, we define Lorentzian reflections with Lorentzian inner product. Then, we examine Lorentzian reflections in view of Lorentzian Householder matrices. Finally, we use pure split quaternions to derive Lorentzian reflections. For each case, we find the matrix representation of Lorentzian reflections and characterize the plane of reflection by using this matrix representation. Moreover, we prove that any Lorentzian orthogonal transformation can be represented by the composition of at most six reflections.

MSC: 15B10, 15A16, 53B30

Keywords: Minkowski space, reflections, rotations

\section{Introduction}

In the Euclidean space, a reflection is an isometry with a hyperplane as a set of fixed points. This set is called the axis (in dimension two) or plane (in dimension three) of reflection. The image of a figure by a reflection is its mirror image in the axis or plane of reflection. A reflection along a subspace in $\mathbb{R}^{n}$ maintains the perpendicular distance from this subspace and its orthogonal complement. And orthogonal complement of this subspace separates the initial and reflected vectors. Thus the components of the initial vector orthogonal to the subspace along which the reflection occurs remain unchanged.

A reflection in $\mathbb{R}^{n}$ can be represented by an $n \times n$ symmetric orthogonal matrix with determinant -1 and eigenvalues $(1,1,1, \ldots 1,-1)$. The product of two such matrices is a special orthogonal matrix which represents a rotation. Every rotation is the result of reflecting in an even number of reflections in hyperplanes through the origin. Thus, the reflections generate orthogonal groups and this result is known as the Cartan-Dieudonné theorem. In [3], answers of the following two frequently asked questions are handled:

Question 1. In how many ways can an orthogonal transformation in an n-dimensional Euclidean space be decomposed?

Question 2. What are the simple reflections that determine a given orthogonal 
transformation in $\mathbb{R}^{n}$ ? Or, in particular "What Householder matrices determine a given orthogonal matrix in $\mathbb{R}^{n}$ ?"

Moreover, a constructive proof of the Cartan Theorem is provided with the relationship of Pythagorean numbers by using Clifford algebras. Then, simple reflections or rotations of canonical vectors are used to generate all Pythagorean vectors and a method is developed to generate the generalized Pythagorean numbers in [4]. On the other hand, some geometrical properties of the Phyagorean quadruples and the rational decomposition of rotations are discussed in [1], and an algorithmic proof of the Cartan-Dieudonné Theorem for spaces with arbitrary signature is given in [9]. Then, Clifford algebras is used to compute the factorization of a given orthogonal transformation as a product of reflections with respect to hyperplanes.

In [6], rotational matrices in Minkowski three-space are generated with unit timelike split quaternions. The geometry of Minkowski four space, which is also called Minkowski space-time, is investigated in more depth in [5]. The eigenvalues and eigenvector of a Lorentzian rotation matrix are examined in term of the coefficients of the corresponding quaternion in [8]. Moreover, the characterizations of eigenvalues (complex or real) of a Lorentzian rotation matrix is also given according to only first component of the corresponding unit timelike quaternion in [8].

This paper is concerned with the reflections and rotations in Minkowski threespace. Firstly, we investigate the Lorentzian reflections by three different approaches. Firstly, we examine Lorentzian reflections with Lorentzian inner product. Then, we express Lorentzian reflections in view of Lorentzian Householder matrices. Finally, we use pure split quaternions to derive Lorentzian reflections. For each case, we find the matrix representation of the Lorentzian reflections and characterize the plane of reflection by using this matrix representation. Moreover, we prove that any Lorentzian orthogonal transformation can be represented by the composition of at most six reflections.

\section{Preliminaries}

In this part, a brief summary of Minkowski three-space and the split quaternions is presented to provide the necessary background.

The Minkowski three-space is a Euclidean three-space, $\mathbb{E}^{3}$, equipped with Lorentzian inner product

$$
\langle\mathbf{u}, \mathbf{v}\rangle_{\mathbb{L}}=-u_{1} v_{1}+u_{2} v_{2}+u_{3} v_{3}
$$


where $\mathbf{u}=\left(u_{1}, u_{2}, u_{3}\right), \mathbf{v}=\left(v_{1}, v_{2}, v_{3}\right) \in \mathbb{E}_{1}^{3}$. The norm of the vector $\mathbf{u} \in \mathbb{E}_{1}^{3}$ is defined by

$$
\|\mathbf{u}\|=\sqrt{\left|\langle\mathbf{u}, \mathbf{u}\rangle_{\mathbb{L}}\right|}
$$

Lorentzian inner product characterizes the elements $\mathbf{u}=\left(u_{1}, u_{2}, u_{3}\right)$ of $\mathbb{E}_{1}^{3}$ as follows:

if $\varepsilon_{\mathbf{u}}=\langle\mathbf{u}, \mathbf{u}\rangle_{\mathbb{L}}>0$ or $\mathbf{u}=0$ then $\mathbf{u}$ is called spacelike

if $\varepsilon_{\mathbf{u}}=\langle\mathbf{u}, \mathbf{u}\rangle_{\mathbb{L}}<0$ then $\mathbf{u}$ is called timelike

if $\varepsilon_{\mathbf{u}}=\langle\mathbf{u}, \mathbf{u}\rangle_{\mathbb{L}}=0$ and $\mathbf{u} \neq 0$ then $\mathbf{u}$ is called lightlike or null.

Proposition 1. Let $\mathbf{u}$ and $\mathbf{v}$ be two non null vectors in $\mathbb{E}_{1}^{3}$ such that $\varepsilon_{\mathbf{u}}=\varepsilon_{\mathbf{v}}$. Then the following properties are satisfied:

i) $(\mathbf{u}-\mathbf{v}) \perp_{\mathbb{L}}(\mathbf{u}+\mathbf{v})$

ii) At least one of the vectors $(\mathbf{u}-\mathbf{v})$ and $(\mathbf{u}+v)$ is a non null vector.

Proof: Suppose that $\mathbf{u}$ and $\mathbf{v}$ are two non null vectors in $\mathbb{E}_{1}^{3}$ such that $\varepsilon_{\mathbf{u}}=\varepsilon_{\mathbf{v}}$.

i)

$$
\langle\mathbf{u}+\mathbf{v}, \mathbf{u}-\mathbf{v}\rangle_{\mathbb{L}}=\varepsilon_{\mathbf{u}}+\langle\mathbf{u}, \mathbf{v}\rangle_{\mathbb{L}}-\langle\mathbf{u}, \mathbf{v}\rangle_{\mathbb{L}}-\varepsilon_{\mathbf{v}}=0
$$

This means that $(\mathbf{u}-\mathbf{v}) \perp_{L}(\mathbf{u}+\mathbf{v})$.

ii) We know that

$$
\begin{aligned}
& \varepsilon_{\mathbf{u}+\mathbf{v}}=\langle\mathbf{u}+\mathbf{v}, \mathbf{u}+\mathbf{v}\rangle_{\mathbb{L}}=2\left(\varepsilon_{\mathbf{u}}+\langle\mathbf{u}, \mathbf{v}\rangle_{\mathbb{L}}\right) \\
& \varepsilon_{\mathbf{u}-\mathbf{v}}=\langle\mathbf{u}-\mathbf{v}, \mathbf{u}-\mathbf{v}\rangle_{\mathbb{L}}=2\left(\varepsilon_{\mathbf{u}}-\langle\mathbf{u}, \mathbf{v}\rangle_{\mathbb{L}}\right) .
\end{aligned}
$$

Using these relations, we have

$$
\varepsilon_{\mathbf{u}+\mathbf{v}}+\varepsilon_{\mathbf{u}-\mathbf{v}}=4 \varepsilon_{\mathbf{u}}=4 \varepsilon_{\mathbf{v}} \neq 0 .
$$

Thus, we get $(\mathbf{u}-\mathbf{v})$ or $(\mathbf{u}+\mathbf{v})$ is a non null vector.

For any $R \in M_{3 \times 3}(\mathbb{R})$, if $\langle R \mathbf{u}, R \mathbf{u}\rangle_{\mathbb{L}}=\langle\mathbf{u}, \mathbf{u}\rangle_{\mathbb{L}}$ for all vectors $\mathbf{u} \in \mathbb{E}_{1}^{3}$, then $R$ is called a semi orthogonal matrix. That is, semi orthogonal matrices preserve the length of vectors in the Minkowski three-space and columns (or rows) of a semi orthogonal matrix form an orthonormal base of $\mathbb{E}_{1}^{3}$. Moreover, $R$ is a semi orthogonal matrix if and only if $I^{*} R^{t} I^{*}=R^{-1}$ where $R^{t}$ is transpose of the matrix $R$ and

$$
I^{*}=\left[\begin{array}{rrr}
-1 & 0 & 0 \\
0 & 1 & 0 \\
0 & 0 & 1
\end{array}\right] \text {. }
$$


So, $R$ is a semi orthogonal matrix if and only if $R^{t} I^{*} R=I^{*}$. Taking the determinant of both sides of this equation gives $\operatorname{det}(R)= \pm 1$. If $\operatorname{det} R=1$, then $R$ is called Lorentzian rotation matrix in $\mathbb{E}_{1}^{3}$. The set of the Lorentzian rotation matrices of $\mathbb{E}_{1}^{3}$ can be expressed as follows

$$
\mathrm{SO}(1,2)=\left\{R \in M_{3 \times 3}(\mathbb{R}) ; R^{t} I^{*} R=I^{*}, \operatorname{det} R=1\right\} .
$$

On the other hand, for any $A \in M_{3 \times 3}(\mathbb{R})$, if $A$ satisfies the equality $I^{*} A^{t} I^{*}=A$, then $A$ is called a semi symmetric matrix in Minkowski three-space. In general, a semi symmetric matrix $A$ can be in the following form

$$
A=\left[\begin{array}{rrr}
a_{11} & a_{12} & a_{13} \\
-a_{12} & a_{22} & a_{23} \\
-a_{13} & a_{23} & a_{33}
\end{array}\right] .
$$

Notice that, the above matrix $A$ satisfies the equality $I^{*} A^{t} I^{*}=A$. If $\operatorname{det} A=-1$, then $A$ is called Lorentzian reflection matrix in $\mathbb{E}_{1}^{3}$.

The set of split quaternions, which was introduced by James Cockle in 1849 [2], can be represented as quadrupoles

$$
\widehat{\mathbb{H}}=\left\{q=q_{0}+q_{1} \mathrm{i}+q_{2} \mathrm{j}+q_{3} \mathrm{k} ; q_{0}, q_{1}, q_{2}, q_{3} \in \mathbb{R}\right\} .
$$

Here the imaginary units satisfy the following relations

$$
\begin{gathered}
\mathrm{i}^{2}=-1, \quad \mathrm{j}^{2}=\mathrm{k}^{2}=\mathrm{ijk}=1 \\
\mathrm{ij}=-\mathrm{ji}=\mathrm{k}, \quad \mathrm{jk}=-\mathrm{kj}=-\mathrm{i}, \quad \mathrm{ki}=-\mathrm{ik}=\mathrm{j} .
\end{gathered}
$$

Unlike quaternion algebra, the set of split quaternions contains zero divisors, nilpotent elements and nontrivial idempotents [7]. For any $q=q_{0}+q_{1} \mathrm{i}+q_{2} \mathrm{j}+q_{3} \mathrm{k} \in \widehat{\mathbb{H}}$, we define scalar part of $q$ as $S_{q}=q_{0}$, vector part of $q$ as $V_{q}=q_{1} \mathrm{i}+q_{2} \mathrm{j}+q_{3} \mathrm{k}$ and conjugate of $q$ as $\bar{q}=S_{q}-V_{q}=q_{0}-q_{1} \mathrm{i}-q_{2} \mathrm{j}-q_{3} \mathrm{k}$. If $S_{q}=0$, then $q$ is called pure split quaternion. And any pure split quaternion $q=q_{1} \mathrm{i}+q_{2} \mathrm{j}+q_{3} \mathrm{k}$ can be represented as $\mathbf{q}=\left(q_{1}, q_{2}, q_{3}\right)$. Furthermore, any pure split quaternion can be considered as a vector in $\mathbb{E}_{1}^{3}$. That is the set of pure split quaternions is identified with Minkowski three-space. The sum and product of two split quaternions $p=p_{0}+p_{1} \mathrm{i}+p_{2} \mathrm{j}+p_{3} \mathrm{k}$ and $q=q_{0}+q_{1} \mathrm{i}+q_{2} \mathrm{j}+q_{3} \mathrm{k}$ are defined as

$$
\begin{aligned}
p+q= & S_{p}+S_{q}+V_{p}+V_{q} \\
= & \left(p_{0}+q_{0}\right)+\left(p_{1}+q_{1}\right) \mathrm{i}+\left(p_{2}+q_{2}\right) \mathrm{j}+\left(p_{3}+q_{3}\right) \mathrm{k} \\
p q= & S_{p} S_{q}+\left\langle V_{p}, V_{q}\right\rangle_{\mathbb{L}}+S_{p} V_{q}+S_{q} V_{p}+V_{p} \times_{\mathbb{L}} V_{q} \\
= & \left(p_{0} q_{0}-p_{1} q_{1}+p_{2} q_{2}+p_{3} q_{3}\right)+\left(p_{1} q_{0}+p_{0} q_{1}-p_{2} q_{3}+p_{3} q_{2}\right) \mathrm{i} \\
& +\left(p_{0} q_{2}+p_{2} q_{0}-p_{1} q_{3}+p_{3} q_{1}\right) \mathrm{j}+\left(p_{0} q_{3}+p_{3} q_{0}-p_{2} q_{1}+p_{1} q_{2}\right) \mathrm{k}
\end{aligned}
$$


respectively. Here $\times_{\mathbb{L}}$ denotes the Lorentzian vector product defined as follows

$$
V_{p} \times_{\mathbb{L}} V_{q}=\left|\begin{array}{ccc}
-\mathrm{i} & \mathrm{j} & \mathrm{k} \\
p_{1} & p_{2} & p_{3} \\
q_{1} & q_{2} & q_{3}
\end{array}\right|
$$

The term $I_{q}=q \bar{q}=\bar{q} q=q_{0}^{2}+q_{1}^{2}-q_{2}^{2}-q_{3}^{2}$ characterizes any split quaternion $q$. The split quaternion $q$ is called spacelike, timelike or null, if $I_{q}<0, I_{q}>0$ or $I_{q}=0$, respectively. And the norm of $q$ is defined as

$$
N_{q}=\sqrt{\left|I_{q}\right|}=\sqrt{\left|q_{0}^{2}+q_{1}^{2}-q_{2}^{2}-q_{3}^{2}\right|}
$$

If $N_{q}=1$, then $q$ is called unit split quaternion. If $I_{q} \neq 0$, then $q^{-1}=\frac{\bar{q}}{I_{q}}[7,8]$.

\section{Lorentzian Reflections}

In this part, we investigate the reflections in Minkowski three-space by three different approach. Firstly, we define Lorentzian reflections with Lorentzian inner product. Then, we examine Lorentzian reflections in view of Lorentzian Householder matrices. Finally, we use pure split quaternions to derive Lorentzian reflections.

\subsection{Reflections by Lorentzian Inner Product}

Let us define a simple reflection in Minkowski three-space by using Lorentzian inner product. Consider the orthogonal complement (plane) of the vector $\mathbf{x}$, defined by

$$
\mathbf{x}^{\perp}=\left\{\mathbf{y} \in \mathbb{E}_{1}^{3} ;\langle\mathbf{x}, \mathbf{y}\rangle_{\mathbb{L}}=0\right\}
$$

For any nonzero and collinear vectors $\mathbf{x}$ and $\mathbf{y}$ in $\mathbb{E}_{1}^{3}$. The projection of a vector $\mathbf{y}$ onto $\mathbf{x}^{\perp}$ is $\mathbf{y}-r \mathbf{x}$ where $r \in \mathbb{R}$ is chosen such that $\mathbf{y}-r \mathbf{x} \in \mathbf{x}^{\perp}$. That is

$$
\langle\mathbf{y}-r \mathbf{x}, \mathbf{x}\rangle_{\mathbb{L}}=0 \Longrightarrow r=\frac{\langle\mathbf{x}, \mathbf{y}\rangle_{\mathbb{L}}}{\varepsilon_{\mathbf{x}}}
$$

where $\mathbf{x}$ is non null and $\varepsilon_{\mathbf{x}}=\langle\mathbf{x}, \mathbf{x}\rangle_{\mathbb{L}}$. In this way, the reflection of $\mathbf{y}$ through the plane $\mathbf{x}^{\perp}$ is called a simple reflection and is given by

$$
\varphi_{\mathbf{x}}(\mathbf{y})=\mathbf{y}-2 r \mathbf{x}=\mathbf{y}-2 \frac{\langle\mathbf{x}, \mathbf{y}\rangle_{\mathbb{L}}}{\varepsilon_{\mathbf{x}}} \mathbf{x}
$$


It is essential to note that if $\mathbf{y} \in \mathbf{x}^{\perp}$, then $\varphi_{\mathbf{x}}(\mathbf{y})=\mathbf{y}$. This means $\varphi_{\mathbf{x}}$ acts as identity map on the plane $\mathbf{x}^{\perp}$. Now, let us show that the inverse of a simple reflection is areflection as well, that is for any $\mathbf{y} \in \mathbb{E}_{1}^{3}$, we have

$$
\begin{aligned}
\varphi_{\mathbf{x}}\left(\varphi_{\mathbf{x}}(\mathbf{y})\right) & =\varphi_{\mathbf{x}}(\mathbf{y}-2 r \mathbf{x})=\mathbf{y}-2 r \mathbf{x}-2 \frac{\langle\mathbf{x}, \mathbf{y}-2 r \mathbf{x}\rangle_{\mathbb{L}}}{\varepsilon_{\mathbf{x}}} \mathbf{x} \\
& =\mathbf{y}-2 r \mathbf{x}-2 \frac{\langle\mathbf{x}, \mathbf{y}\rangle_{\mathbb{L}}-2 r\langle\mathbf{x}, \mathbf{x}\rangle_{\mathbb{L}}}{\varepsilon_{\mathbf{x}}} \mathbf{x}=\mathbf{y}-2 r \mathbf{x}-2 r \mathbf{x}+4 r \mathbf{x}=\mathbf{y} .
\end{aligned}
$$

Moreover, $\varphi_{\mathbf{x}}$ is an orthogonal transformation. Since

$$
\begin{aligned}
\left\|\varphi_{\mathbf{x}}(\mathbf{y})\right\|^{2} & =\langle\mathbf{y}-2 r \mathbf{x}, \mathbf{y}-2 r \mathbf{x}\rangle_{\mathbb{L}}=\langle\mathbf{y}, \mathbf{y}\rangle_{\mathbb{L}}-4 r\langle\mathbf{x}, \mathbf{y}\rangle_{\mathbb{L}}+4 r^{2}\langle\mathbf{x}, \mathbf{x}\rangle_{\mathbb{L}} \\
& =\langle\mathbf{y}, \mathbf{y}\rangle_{\mathbb{L}}-\frac{4 r}{\varepsilon_{\mathbf{x}}}\left(\langle\mathbf{x}, \mathbf{y}\rangle_{\mathbb{L}}\right)^{2}+\frac{4 r}{\varepsilon_{\mathbf{x}}}\left(\langle\mathbf{x}, \mathbf{x}\rangle_{\mathbb{L}}\right)^{2}=\langle\mathbf{y}, \mathbf{y}\rangle_{\mathbb{L}}=\|\mathbf{y}\|^{2}
\end{aligned}
$$

Theorem 2. For any two non null vectors $\mathbf{u}$ and $\mathbf{v}$ in $\mathbb{E}_{1}^{3}$, such that $\mathbf{u} \neq \mathbf{v}$ and $\varepsilon_{\mathbf{u}}=\varepsilon_{\mathbf{v}}$, there exists a unique Lorentzian reflection or rotation such that $\varphi(\mathbf{u})=$ v.

Proof: Let $\mathbf{u}$ and $\mathbf{v}$ be two non null different vectors in $\mathbb{E}_{1}^{3}$ such that $\varepsilon_{\mathbf{u}}=\varepsilon_{\mathbf{v}}$. Consider the map $\varphi: \mathbb{E}_{1}^{3} \rightarrow \mathbb{E}_{1}^{3}$ defined as

$$
\varphi(\mathbf{x})=\left\{\begin{aligned}
\varphi_{\mathbf{u}}(\mathbf{x}) & \text { if } \mathbf{v}=-\mathbf{u} \\
\varphi_{\mathbf{u}-\mathbf{v}}(\mathbf{x}) & \text { if } \mathbf{v} \neq-\mathbf{u} \text { and }(\mathbf{u}-\mathbf{v}) \text { is non null } \\
-\varphi_{\mathbf{u}+\mathbf{v}}(\mathbf{x}) & \text { if } \mathbf{v} \neq-\mathbf{u} \text { and }(\mathbf{u}-\mathbf{v}) \text { is null. }
\end{aligned}\right.
$$

If $\mathbf{v}=-\mathbf{u}$, then

$$
\varphi(\mathbf{u})=\varphi_{\mathbf{u}}(\mathbf{u})=\mathbf{u}-2 \frac{\langle\mathbf{u}, \mathbf{u}\rangle_{\mathbb{L}}}{\langle\mathbf{u}, \mathbf{u}\rangle_{\mathbb{L}}} \mathbf{u}=\mathbf{u}-2 \mathbf{u}=-\mathbf{u}=\mathbf{v} .
$$

If $\mathbf{v} \neq-\mathbf{u}$ and $(\mathbf{u}-\mathbf{v})$ is a non null vector, then

$$
\begin{aligned}
\varphi(\mathbf{u}) & =\varphi_{\mathbf{u}-\mathbf{v}}(\mathbf{u})=\mathbf{u}-2 \frac{\langle\mathbf{u}, \mathbf{u}-\mathbf{v}\rangle_{\mathbb{L}}}{\langle\mathbf{u}-\mathbf{v}, \mathbf{u}-\mathbf{v}\rangle_{\mathbb{L}}}(\mathbf{u}-\mathbf{v}) \\
& =\mathbf{u}-2 \frac{\langle\mathbf{u}, \mathbf{u}\rangle_{\mathbb{L}}-\langle\mathbf{u}, \mathbf{v}\rangle_{\mathbb{L}}}{2\left(\langle\mathbf{u}, \mathbf{u}\rangle_{\mathbb{L}}-\langle\mathbf{u}, \mathbf{v}\rangle_{\mathbb{L}}\right)}(\mathbf{u}-\mathbf{v})=\mathbf{u}-(\mathbf{u}-\mathbf{v})=\mathbf{v} .
\end{aligned}
$$

If $\mathbf{v} \neq-\mathbf{u}$ and $(\mathbf{u}-\mathbf{v})$ is a null vector, then

$$
\begin{aligned}
\varphi(\mathbf{u}) & =-\varphi_{\mathbf{u}+\mathbf{v}}(\mathbf{u})=-\mathbf{u}+2 \frac{\langle\mathbf{u}, \mathbf{u}+\mathbf{v}\rangle_{\mathbb{L}}}{\langle\mathbf{u}+\mathbf{v}, \mathbf{u}+\mathbf{v}\rangle_{\mathbb{L}}}(\mathbf{u}+\mathbf{v}) \\
& =-\mathbf{u}+2 \frac{\langle\mathbf{u}, \mathbf{u}\rangle_{\mathbb{L}}+\langle\mathbf{u}, \mathbf{v}\rangle_{\mathbb{L}}}{2\left(\langle\mathbf{u}, \mathbf{u}\rangle_{\mathbb{L}}+\langle\mathbf{u}, \mathbf{v}\rangle_{\mathbb{L}}\right)}(\mathbf{u}+\mathbf{v})=-\mathbf{u}+(\mathbf{u}+\mathbf{v})=\mathbf{v} .
\end{aligned}
$$


By above discussions and construction of the map $\varphi$, the simple reflection or rotation is unique such that $\varphi(\mathbf{u})=\mathbf{v}$.

Remark 3. The case $\mathbf{u}=\mathbf{v}$ is special. Any plane that contains the vector a determines a simple reflection that satisfies $\varphi(\mathbf{u})=\mathbf{u}$. Therefore, in general, there are infinitely many different simple reflections that leave $\mathbf{u}$ fixed. For this reason, in the remainder of this paper, for the case $\mathbf{u}=\mathbf{v}$ we will take $\varphi$ as the identity operator instead of taking a simple reflection.

Theorem 4. The matrix representation of Lorentzian reflection $\varphi_{\mathbf{x}}$ through the hyperplane $\mathrm{x}^{\perp}$ is obtained as follows

$$
\varphi_{\mathbf{x}}=\frac{1}{\varepsilon_{\mathbf{x}}}\left[\begin{array}{ccc}
x_{1}^{2}+x_{2}^{2}+x_{3}^{2} & -2 x_{1} x_{2} & -2 x_{1} x_{3} \\
2 x_{1} x_{2} & -x_{1}^{2}-x_{2}^{2}+x_{3}^{2} & -2 x_{2} x_{3} \\
2 x_{1} x_{3} & -2 x_{2} x_{3} & -x_{1}^{2}+x_{2}^{2}-x_{3}^{2}
\end{array}\right]
$$

where $\mathbf{x}=\left(x_{1}, x_{2}, x_{3}\right)$.

Proof: Let $\left\{\mathbf{e}_{1}, \mathbf{e}_{2}, \mathbf{e}_{3}\right\}$ be the standard basis of $\mathbb{E}_{1}^{3}$ and $\mathbf{x}=\left(x_{1}, x_{2}, x_{3}\right)$. Then we have

$$
\begin{aligned}
& \varphi_{\mathbf{x}}\left(\mathbf{e}_{1}\right)=\mathbf{e}_{1}-2 \frac{\left\langle\mathbf{x}, \mathbf{e}_{1}\right\rangle_{L}}{\varepsilon_{\mathbf{x}}} \mathbf{x}=\left(1+2 \frac{x_{1}^{2}}{\varepsilon_{\mathbf{x}}}, 2 x_{1} \frac{x_{2}}{\varepsilon_{\mathbf{x}}}, 2 x_{1} \frac{x_{3}}{\varepsilon_{\mathbf{x}}}\right) \\
& \varphi_{\mathbf{x}}\left(\mathbf{e}_{2}\right)=\mathbf{e}_{2}-2 \frac{\left\langle\mathbf{x}, \mathbf{e}_{2}\right\rangle_{L}}{\varepsilon_{\mathbf{x}}} \mathbf{x}=\left(-2 x_{1} \frac{x_{2}}{\varepsilon_{\mathbf{x}}}, 1-2 \frac{x_{2}^{2}}{\varepsilon_{\mathbf{x}}},-2 x_{2} \frac{x_{3}}{\varepsilon_{\mathbf{x}}}\right) \\
& \varphi_{\mathbf{x}}\left(\mathbf{e}_{2}\right)=\mathbf{e}_{3}-2 \frac{\left\langle\mathbf{x}, \mathbf{e}_{3}\right\rangle_{L}}{\varepsilon_{\mathbf{x}}} \mathbf{x}=\left(-2 x_{1} \frac{x_{3}}{\varepsilon_{\mathbf{x}}},-2 x_{2} \frac{x_{3}}{\varepsilon_{\mathbf{x}}}, 1-2 \frac{x_{3}^{2}}{\varepsilon_{\mathbf{x}}}\right) .
\end{aligned}
$$

So we get the matrix representation of the Lorentzian reflection as started above.

Notice that the matrix $\varphi_{\mathbf{x}}$ has determinant -1 and satisfies the relation $I^{*} \varphi_{\mathbf{x}} I^{*}=$ $\varphi_{\mathbf{x}}^{t}$. And the Lorentzian reflection $\varphi_{\mathbf{x}}$ through the hyperplane $\mathbf{x}^{\perp}$ has the set of eigenvalues $\{-1,1,1\}$ with the corresponding eigenvectors

$$
\left\{\left(x_{1}, x_{2}, x_{3}\right),\left(x_{2}, x_{1}, 0\right),\left(x_{3}, 0, x_{1}\right)\right\}
$$

In other words, the plane of reflection can be determined by the eigenvector of the Lorentzian reflection matrix corresponding to the eigenvalue -1 . 


\subsection{Reflections by Lorentzian Householder Transformation}

Let $I$ be $3 \times 3$ identity matrix and $I^{*}=\operatorname{diag}\{-1,1,1\}$. The Lorentzian Householder transformation associated with the non null vector $\mathbf{x}=\left(x_{1}, x_{2}, x_{3}\right) \in \mathbb{E}_{1}^{3}$ is defined as

$$
H_{\mathbf{x}}=I-\frac{2}{\varepsilon_{\mathbf{x}}} \mathbf{x x}^{t} I^{*} \equiv \varphi_{\mathbf{x}}
$$

It can be easily seen that Lorentzian Householder transformation $H_{\mathbf{x}}$ has the following properties:

- it is semi symmetric: $I^{*} H_{\mathbf{x}} I^{*}=H^{t}$

- it is semi orthogonal: $I^{*} H_{\mathrm{x}}^{-1} I^{*}=H^{t}$

- hence it is involutory: $H_{\mathbf{x}}^{2}=I$.

Moreover, $H_{\mathbf{x}}$ has the set of eigenvalues $\{-1,1,1\}$ with the corresponding eigenvectors $\left\{\left(x_{1}, x_{2}, x_{3}\right),\left(x_{2}, x_{1}, 0\right),\left(x_{3}, 0, x_{1}\right)\right\}$, respectively. The determinant of $H_{\mathbf{x}}$ is -1 , since the determinant of a matrix is the product of its eigenvalues. Therefore, Lorentzian Householder transformation $H_{\mathrm{x}}$ represents the Lorentzian reflection where the hyperplane of the reflection is $x^{\perp}$. The reflection of a point $\mathbf{u} \in \mathbb{E}_{1}^{3}$ about this hyperplane is

$$
H_{\mathbf{x}}(\mathbf{u})=\mathbf{u}-\frac{2}{\varepsilon_{\mathbf{x}}} \mathbf{x x}^{t} I^{*} \mathbf{u}
$$

Theorem 5. For any two non null vectors $\mathbf{u}$ and $\mathbf{v}$ in $\mathbb{E}_{1}^{3}$, such that $\mathbf{u} \neq \mathbf{v}$ and $\varepsilon_{\mathbf{u}}=$ $\varepsilon_{\mathbf{v}}$, there exists a unique Lorentzian Householder transformation or composition of two Householder transformation $H$ such that $H(\mathbf{u})=\mathbf{v}$.

Proof: Let $\mathbf{u}$ and $\mathbf{v}$ be two non null different vectors in $\mathbb{E}_{1}^{3}$ such that $\varepsilon_{\mathbf{u}}=\varepsilon_{\mathbf{v}}$. Consider the map $H: \mathbb{E}_{1}^{3} \rightarrow \mathbb{E}_{1}^{3}$ defined as

$$
H(\mathbf{x})=\left\{\begin{aligned}
H_{\mathbf{u}}(\mathbf{x}) & \text { if } \mathbf{v}=-\mathbf{u} \\
H_{\mathbf{u}-\mathbf{v}}(\mathbf{x}) & \text { if } \mathbf{v} \neq-\mathbf{u} \text { and }(\mathbf{u}-\mathbf{v}) \text { is non null } \\
-H_{\mathbf{u}+\mathbf{v}}(\mathbf{x}) & \text { if } \mathbf{v} \neq-\mathbf{u} \text { and }(\mathbf{u}-\mathbf{v}) \text { is null. }
\end{aligned}\right.
$$

Then, if $\mathbf{v}=-\mathbf{u}$, then

$$
H(\mathbf{u})=H_{\mathbf{u}}(\mathbf{u})=\mathbf{u}-\frac{2}{\varepsilon_{\mathbf{u}}} \mathbf{u u}^{t} I^{*} \mathbf{u}=\mathbf{u}-\frac{2}{\varepsilon_{\mathbf{u}}} \mathbf{u}\left(\varepsilon_{\mathbf{u}}\right)=-\mathbf{u}=\mathbf{v} .
$$


If $\mathbf{v} \neq-\mathbf{u}$ and $(\mathbf{u}-\mathbf{v})$ is a non null vector, then we get

$$
\begin{aligned}
H(\mathbf{u}) & =H_{\mathbf{u}-\mathbf{v}}(\mathbf{u})=\mathbf{u}-\frac{2}{\varepsilon_{\mathbf{u}-\mathbf{v}}}(\mathbf{u}-\mathbf{v})(\mathbf{u}-\mathbf{v})^{t} I^{*} \mathbf{u} \\
& =\mathbf{u}-\frac{2\langle\mathbf{u}, \mathbf{u}\rangle_{\mathbb{L}}-\langle\mathbf{u}, \mathbf{v}\rangle_{\mathbb{L}}}{2\left(\langle\mathbf{u}, \mathbf{u}\rangle_{\mathbb{L}}-\langle\mathbf{u}, \mathbf{v}\rangle_{\mathbb{L}}\right)}(\mathbf{u}-\mathbf{v})=\mathbf{u}-(\mathbf{u}-\mathbf{v})=\mathbf{v}
\end{aligned}
$$

by using the identities $\mathbf{u}^{t} I^{*} \mathbf{u}=\mathbf{v}^{t} I^{*} \mathbf{v}=\varepsilon_{\mathbf{u}}=\varepsilon_{\mathbf{v}}$ and $\mathbf{u}^{t} I^{*} \mathbf{v}=\mathbf{v}^{t} I^{*} \mathbf{u}=\langle\mathbf{u}, \mathbf{v}\rangle_{\mathbb{L}}$. If $\mathbf{v} \neq-\mathbf{u}$ and $(\mathbf{u}-\mathbf{v})$ is a null vector, then we obtain

$$
\begin{aligned}
& H(\mathbf{u})=-H_{\mathbf{u}+\mathbf{v}}(\mathbf{u})=-\mathbf{u}+\frac{2}{\varepsilon_{\mathbf{u}+\mathbf{v}}}(\mathbf{u}+\mathbf{v})(\mathbf{u}+\mathbf{v})^{t} I^{*} \mathbf{u} \\
& =-\mathbf{u}+\frac{2\left(\langle\mathbf{u}, \mathbf{u}\rangle_{\mathbb{L}}+\langle\mathbf{u}, \mathbf{v}\rangle_{\mathbb{L}}\right)}{2\left(\langle\mathbf{u}, \mathbf{u}\rangle_{\mathbb{L}}+\langle\mathbf{u}, \mathbf{v}\rangle_{\mathbb{L}}\right)}(\mathbf{u}+\mathbf{v})=-\mathbf{u}+(\mathbf{u}+\mathbf{v})=\mathbf{v} .
\end{aligned}
$$

By above discussions and construction of the map $H$, the Lorentzian Householder transformation $H$ is unique such that $H(\mathbf{u})=\mathbf{v}$.

\subsection{Lorentzian Reflections by Pure Split Quaternions}

Let us define a simple reflection in Minkowski three-space by pure split quaternion. The set of pure split quaternions is denoted by

$$
\operatorname{Im} \widehat{\mathbb{H}}=\left\{q=q_{1} \mathrm{i}+q_{2} \mathrm{j}+q_{3} \mathrm{k} \in \mathbb{H}\right\} .
$$

Since Im $\widehat{\mathbb{H}}$ is identified with Minkowski three-space, then any $x=x_{1} \mathrm{i}+x_{2} \mathrm{j}+$ $x_{3} \mathrm{k} \in \operatorname{Im} \widehat{\mathbb{H}}$ can be considered as a vector $\mathbf{x}=\left(x_{1}, x_{2}, x_{3}\right) \in \mathbb{E}_{1}^{3}$. Now, consider the linear transformation

$$
\phi_{x}: \operatorname{Im} \widehat{\mathbb{H}} \rightarrow \operatorname{Im} \widehat{\mathbb{H}}, \quad u \rightarrow \phi_{x}(u)=-\frac{1}{x^{2}} x u x
$$

where $x$ is a non null pure split quaternion. Let $\mathrm{i}=(1,0,0), \mathrm{j}=(0,1,0), \mathrm{k}=$ $(0,0,1)$ be the standard basis of $\mathbb{E}_{1}^{3}$. Then we have

$$
\begin{aligned}
\phi_{x}(\mathrm{i}) & =\frac{1}{x^{2}}\left(\left(x_{1}^{2}+x_{2}^{2}+x_{3}^{2}\right) \mathrm{i}+\left(2 x_{1} x_{2}\right) \mathrm{j}+\left(2 x_{1} x_{3}\right) \mathrm{k}\right) \\
\phi_{x}(\mathrm{j}) & \left.=\frac{1}{x^{2}}\left(\left(-2 x_{1} x_{2}\right) \mathrm{i}+\left(-x_{1}^{2}-x_{2}^{2}+x_{3}^{2}\right) \mathrm{j}+\left(-2 x_{2} x_{3}\right) \mathrm{k}\right)\right) \\
\phi_{x}(\mathrm{k}) & \left.=\frac{1}{x^{2}}\left(\left(-2 x_{1} x_{3}\right) \mathrm{i}+\left(2 x_{2} x_{3}\right) \mathrm{j}+\left(-x_{1}^{2}+x_{2}^{2}-x_{3}^{2}\right) \mathrm{k}\right)\right) .
\end{aligned}
$$

The matrix representation of the transformation $\phi_{\mathbf{x}}$ is obtained as follows

$$
\phi_{\mathbf{x}}=\frac{1}{x^{2}}\left[\begin{array}{ccc}
x_{1}^{2}+x_{2}^{2}+x_{3}^{2} & -2 x_{1} x_{2} & -2 x_{1} x_{3} \\
2 x_{1} x_{2} & -x_{1}^{2}-x_{2}^{2}+x_{3}^{2} & -2 x_{2} x_{3} \\
2 x_{1} x_{3} & -2 x_{2} x_{3} & -x_{1}^{2}+x_{2}^{2}-x_{3}^{2}
\end{array}\right] .
$$


We can easily see that the linear transformation $\phi_{x}$ satisfies the relations $\phi_{x}^{t} I^{*} \phi_{x}=$ $I^{*}$ and $\operatorname{det} \phi_{x}=-1$. And $\phi_{x}$ has the set of eigenvalues $\{-1,1,1\}$ with the corresponding eigenvectors $\left\{\left(x_{1}, x_{2}, x_{3}\right),\left(x_{2}, x_{1}, 0\right),\left(x_{3}, 0, x_{1}\right)\right\}$, respectively. Therefore, the linear transformation $\phi_{\mathbf{x}}$ represents the Lorentzian reflection where the hyperplane of the reflection is $\mathbf{x}^{\perp}$.

Theorem 6. For any two non null pure split quaternions $u$ and $v$, such that $u \neq v$ and $u^{2}=v^{2}$, there exists a unique Lorentzian reflection or rotation $\phi$ such that $\phi(u)=v$.

Proof: Let $u$ and $v$ be two non null pure split quaternions such that $u^{2}=v^{2}$. Consider the map $\phi: \operatorname{Im} \widehat{\mathbb{H}} \rightarrow \operatorname{Im} \widehat{\mathbb{H}}$ defined as

$$
\phi(x)=\left\{\begin{aligned}
\phi_{u}(x) & \text { if } v=-u \\
\phi_{u-v}(x) & \text { if } v \neq-u \text { and }(u-v) \text { is non null } \\
-\phi_{u+v}(\mathbf{x}) & \text { if } v \neq-u \text { and }(u-v) \text { is null. }
\end{aligned}\right.
$$

If $v=-u$, then we get

$$
\phi(u)=\phi_{u}(u)=-\frac{1}{u^{2}} u u u=-u=v .
$$

If $v \neq-u$ and $(u-v)$ is a non null vector, then we get

$$
\phi(u)=\phi_{u-v}(u)=-\frac{1}{(u-v)^{2}}(u-v) u(u-v)=\frac{\left(2 v^{2}-u v-v u\right)}{\left(2 v^{2}-u v-v u\right)} v=v
$$

by using the identities $u^{2}=v^{2}$ and $2 v^{2}-u v-v u \in \mathbb{R}$. If $\mathbf{v} \neq-\mathbf{u}$ and $(\mathbf{u}-\mathbf{v})$ is a null vector, then we obtain

$$
\phi(u)=\phi_{u+v}(u)=-\frac{1}{(u+v)^{2}}(u+v) u(u+v)=\frac{\left(2 v^{2}+u v+v u\right)}{\left(2 v^{2}+u v+v u\right)} v=v .
$$

By above discussions and construction of the map $\phi$, the Lorentzian reflection or rotation $\phi$ is unique such that $\phi(u)=v$.

\section{Lorentzian Rotations and Cartan-Dieudonné Theorem}

Theorem 7. Product of two Lorentzian reflection matrices is a Lorentzian rotation matrix.

Proof: Let $\varphi_{\mathbf{x}}$ and $\varphi_{\mathbf{y}}$ be two Lorentzian reflection matrices with the planes of reflection $x^{\perp}$ and $\mathbf{y}^{\perp}$, respectively. Since $\operatorname{det} \varphi_{\mathbf{x}}=\operatorname{det} \varphi_{\mathbf{y}}-1$, then $R=\varphi_{\mathbf{x}} \varphi_{\mathbf{y}}$ 
must have determinant 1 . Now, we only need to show that $R^{t} I^{*} R=I^{*}$. By using the identities $\varphi_{\mathbf{x}}^{t} I^{*}=I^{*} \varphi_{\mathbf{x}}, \varphi_{\mathbf{y}}^{t} I^{*}=I^{*} \varphi_{\mathbf{y}}, \varphi_{\mathbf{x}}^{-1}=\varphi_{\mathbf{x}}$ and $\varphi_{\mathbf{y}}^{-1}=\varphi_{\mathbf{y}}$, we may write

$$
\begin{aligned}
R^{t} I^{*} R=\left(\varphi_{\mathbf{x}} \varphi_{\mathbf{y}}\right)^{t} I^{*}\left(\varphi_{\mathbf{x}} \varphi_{\mathbf{y}}\right)=\varphi_{\mathbf{y}}{ }^{t} & \left(\varphi_{\mathbf{x}}^{t} I^{*}\right) \varphi_{\mathbf{x}} \varphi_{\mathbf{y}} \\
& =\left(\varphi_{\mathbf{y}}{ }^{t} I^{*}\right)\left(\varphi_{\mathbf{x}} \varphi_{\mathbf{x}}\right) \varphi_{\mathbf{y}}=I^{*} \varphi_{\mathbf{y}} \varphi_{\mathbf{y}}=I^{*}
\end{aligned}
$$

Theorem 8. For any two non null vectors $\mathbf{u}$ and $\mathbf{v}$ in $\mathbb{E}_{1}^{3}$, such that $\mathbf{u} \neq \mathbf{v}$ and $\varepsilon_{\mathbf{u}}=\varepsilon_{\mathbf{v}}$, there exists a unique rotation or reflection such that $R(\mathbf{u})=\mathbf{v}$.

Proof: Let $\mathbf{u}$ and $\mathbf{v}$ be two non null different vectors in $\mathbb{E}_{1}^{3}$ such that $\varepsilon_{\mathbf{u}}=\varepsilon_{\mathbf{v}}$. Consider the map $R: \mathbb{E}_{1}^{3} \rightarrow \mathbb{E}_{1}^{3}$ defined as

$$
R(\mathbf{x})=\left\{\begin{aligned}
-\varphi_{\mathbf{v}}\left(\varphi_{\mathbf{u}}(\mathbf{x})\right) & \text { if } \mathbf{v}=-\mathbf{u} \\
-\varphi_{\mathbf{v}}\left(\varphi_{\mathbf{u}-\mathbf{v}}(\mathbf{x})\right) & \text { if } \mathbf{v} \neq-\mathbf{u} \text { and }(\mathbf{u}-\mathbf{v}) \text { is non null } \\
\varphi_{\mathbf{v}}\left(\varphi_{\mathbf{u}+\mathbf{v}}(\mathbf{x})\right) & \text { if } \mathbf{v} \neq-\mathbf{u} \text { and }(\mathbf{u}-\mathbf{v}) \text { is null. }
\end{aligned}\right.
$$

If $\mathbf{v}=-\mathbf{u}$, then

$$
\varphi_{\mathbf{u}}(\mathbf{u})=\mathbf{u}-2 \frac{\langle\mathbf{u}, \mathbf{u}\rangle_{\mathbb{L}}}{\langle\mathbf{u}, \mathbf{u}\rangle_{\mathbb{L}}} \mathbf{u}=\mathbf{u}-2 \mathbf{u}=-\mathbf{u}=\mathbf{v}
$$

and

$$
R(\mathbf{u})=-\varphi_{\mathbf{v}}\left(\varphi_{\mathbf{u}}(\mathbf{u})\right)=-\varphi_{\mathbf{v}}(\mathbf{v})=-\mathbf{v}+2 \frac{\langle\mathbf{v}, \mathbf{v}\rangle_{\mathbb{L}}}{\langle\mathbf{v}, \mathbf{v}\rangle_{\mathbb{L}}} \mathbf{v}=\mathbf{v}
$$

If $\mathbf{v} \neq-\mathbf{u}$ and $(\mathbf{u}-\mathbf{v})$ is a non null vector, then

$$
\begin{aligned}
\varphi_{\mathbf{u}-\mathbf{v}}(\mathbf{u}) & =\mathbf{u}-2 \frac{\langle\mathbf{u}, \mathbf{u}-\mathbf{v}\rangle_{\mathbb{L}}}{\langle\mathbf{u}-\mathbf{v}, \mathbf{u}-\mathbf{v}\rangle_{\mathbb{L}}}(\mathbf{u}-\mathbf{v}) \\
& =\mathbf{u}-2 \frac{\langle\mathbf{u}, \mathbf{u}\rangle_{\mathbb{L}}-\langle\mathbf{u}, \mathbf{v}\rangle_{\mathbb{L}}}{2\left(\langle\mathbf{u}, \mathbf{u}\rangle_{\mathbb{L}}-\langle\mathbf{u}, \mathbf{v}\rangle_{\mathbb{L}}\right)}(\mathbf{u}-\mathbf{v})=\mathbf{u}-(\mathbf{u}-\mathbf{v})=\mathbf{v}
\end{aligned}
$$

and

$$
R(\mathbf{u})=-\varphi_{\mathbf{v}}\left(\varphi_{\mathbf{u}-\mathbf{v}}(\mathbf{u})\right)=-\varphi_{\mathbf{v}}(\mathbf{v})=\mathbf{v}
$$

and if $\mathbf{v} \neq-\mathbf{u}$ and $(\mathbf{u}-\mathbf{v})$ is a null vector, then

$$
\begin{aligned}
\varphi_{\mathbf{u}+\mathbf{v}}(\mathbf{u}) & =\mathbf{u}-2 \frac{\langle\mathbf{u}, \mathbf{u}+\mathbf{v}\rangle_{\mathbb{L}}}{\langle\mathbf{u}+\mathbf{v}, \mathbf{u}+\mathbf{v}\rangle_{\mathbb{L}}}(\mathbf{u}+\mathbf{v}) \\
& =\mathbf{u}-2 \frac{\langle\mathbf{u}, \mathbf{u}\rangle_{\mathbb{L}}+\langle\mathbf{u}, \mathbf{v}\rangle_{\mathbb{L}}}{2\left(\langle\mathbf{u}, \mathbf{u}\rangle_{\mathbb{L}}+\langle\mathbf{u}, \mathbf{v}\rangle_{\mathbb{L}}\right)}(\mathbf{u}+\mathbf{v})=\mathbf{u}-(\mathbf{u}+\mathbf{v})=-\mathbf{v}
\end{aligned}
$$


and

$$
R(\mathbf{u})=\varphi_{\mathbf{v}}\left(\varphi_{\mathbf{u}+\mathbf{v}}(\mathbf{u})\right)=\varphi_{\mathbf{v}}(-\mathbf{v})=\mathbf{v}
$$

By above discussions and construction of the map $R$, the Lorentzian rotation is unique such that $R(\mathbf{u})=\mathbf{v}$.

Theorem 9. Any orthogonal transformation of $\mathbb{E}_{1}^{3}$ can be written as a decomposition of at most six Lorentzian reflections.

Proof: We will apply Theorem 2 recurrently at each step. Let $R: \mathbb{E}_{1}^{3} \rightarrow \mathbb{E}_{1}^{3}$ be an orthogonal transformation and let $\left\{\mathbf{e}_{1}, \mathbf{e}_{2}, \mathbf{e}_{3}\right\}$ be an orthogonal basis for $\mathbb{E}_{1}^{3}$.

Step 1: First consider $\mathbf{v}=\mathbf{e}_{1}$ and $\mathbf{u}=R\left(\mathbf{e}_{1}\right)$. Since $R$ is an orthogonal transformation, we have $\varepsilon_{\mathbf{u}}=\varepsilon_{\mathbf{v}}$. If $R\left(\mathbf{e}_{1}\right) \neq \mathbf{e}_{1}$, according to Theorem 2 , there exists a simple reflection

$$
\varphi_{1}(\mathbf{x})=\left\{\begin{aligned}
\varphi_{\mathbf{e}_{1}}(\mathbf{x}) & \text { if } R\left(\mathbf{e}_{1}\right)=-\mathbf{e}_{1} \\
\varphi_{R\left(\mathbf{e}_{1}\right)-\mathbf{e}_{1}}(\mathbf{x}) & \text { if } R\left(\mathbf{e}_{1}\right) \neq-\mathbf{e}_{1} \text { and } R\left(\mathbf{e}_{1}\right)-\mathbf{e}_{1} \text { is non null } \\
-\varphi_{R\left(\mathbf{e}_{1}\right)+\mathbf{e}_{1}}(\mathbf{x}) & \text { if } R\left(\mathbf{e}_{1}\right) \neq-\mathbf{e}_{1} \text { and } R\left(\mathbf{e}_{1}\right)-\mathbf{e}_{1} \text { is null }
\end{aligned}\right.
$$

where $\mathbf{x}_{1}=R\left(\mathbf{e}_{1}\right)-\mathbf{e}_{1}$, that satisfies $\varphi_{1}\left(R\left(\mathbf{e}_{1}\right)\right)=\mathbf{e}_{1}$. Since $\varphi_{1} \circ R$ is an orthogonal transformation, for $i=2,3$ we have that $\varphi_{1}\left(R\left(\mathbf{e}_{i}\right)\right)$ is orthogonal to $\varphi_{1}\left(R\left(\mathbf{e}_{1}\right)\right)=\mathbf{e}_{1}$. Therefore

$$
\varphi_{1}\left(R\left(\mathbf{e}_{i}\right)\right) \in \operatorname{Span}\left\{\mathbf{e}_{2}, \mathbf{e}_{3}\right\} .
$$

If it turns out that $R\left(\mathbf{e}_{1}\right)=\mathbf{e}_{1}$, then, according to Remark 3 we take $\varphi_{1}$ as the identity operator. We will do the same in the corresponding case of subsequent steps of the procedure.

Step 2: Now consider $\mathbf{v}=\mathbf{e}_{2}$ and $\mathbf{u}=\varphi_{1}\left(R\left(\mathbf{e}_{2}\right)\right)$. If $\varphi_{1}\left(R\left(\mathbf{e}_{2}\right)\right) \neq \mathbf{e}_{2}$ then, application of Theorem 2 again yields the simple reflection

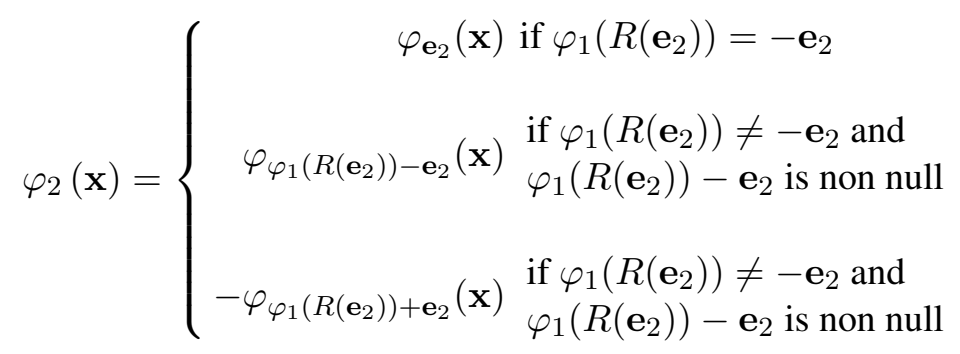

where $\mathbf{x}_{2}=\varphi_{1}\left(R\left(\mathbf{e}_{2}\right)\right)-\mathbf{e}_{2}$, that satisfies $\varphi_{2}\left(\varphi_{1}\left(R\left(\mathbf{e}_{2}\right)\right)\right)=\mathbf{e}_{2}$. Since $\varphi_{1} \circ R$ is an orthogonal transformation, we have $\varphi_{1}\left(R\left(\mathbf{e}_{1}\right)\right)=\mathbf{e}_{1}$ is orthogonal to $\varphi_{1}\left(R\left(\mathbf{e}_{2}\right)\right)$. Hence $\mathbf{e}_{1} \in \mathbf{x}_{2}^{\perp}$ then $\varphi_{2}\left(\mathbf{e}_{1}\right)=\mathbf{e}_{1}$. Therefore, we have

$$
\varphi_{2}\left(\varphi_{1}\left(R\left(\mathbf{e}_{i}\right)\right)\right)=\mathbf{e}_{i} \text { for } i=1,2, \quad \varphi_{2}\left(\varphi_{1}\left(R\left(\mathbf{e}_{3}\right)\right)\right) \in \operatorname{Span}\left\{\mathbf{e}_{3}\right\} .
$$


Step 3: Now consider $\mathbf{v}=\mathbf{e}_{3}$ and $\mathbf{u}=\varphi_{2}\left(\varphi_{1}\left(R\left(\mathbf{e}_{3}\right)\right)\right)$. If $\varphi_{2}\left(\varphi_{1}\left(R\left(\mathbf{e}_{3}\right)\right)\right) \neq \mathbf{e}_{3}$ then, application of Theorem 2 again yields the simple reflection

$$
\varphi_{3}(\mathbf{x})=\left\{\begin{array}{cl}
\varphi_{\mathbf{e}_{3}}(\mathbf{x}) \text { if } \varphi_{2}\left(\varphi_{1}\left(R\left(\mathbf{e}_{3}\right)\right)\right)=-\mathbf{e}_{3} \\
\varphi_{\varphi_{2}\left(\varphi_{1}\left(R\left(\mathbf{e}_{3}\right)\right)\right)-\mathbf{e}_{3}(\mathbf{x})} \begin{array}{l}
\text { if } \varphi_{2}\left(\varphi_{1}\left(R\left(\mathbf{e}_{3}\right)\right)\right) \neq-\mathbf{e}_{3} \text { and } \\
\varphi_{2}\left(\varphi_{1}\left(R\left(\mathbf{e}_{3}\right)\right)\right)-\mathbf{e}_{3} \text { is non null }
\end{array} \\
-\varphi_{\varphi_{2}\left(\varphi_{1}\left(R\left(\mathbf{e}_{3}\right)\right)\right)+\mathbf{e}_{3}(\mathbf{x})} \begin{array}{l}
\text { if } \varphi_{2}\left(\varphi_{1}\left(R\left(\mathbf{e}_{3}\right)\right)\right) \neq-\mathbf{e}_{3} \text { and } \\
\varphi_{2}\left(\varphi_{1}\left(R\left(\mathbf{e}_{3}\right)\right)\right)-\mathbf{e}_{3} \text { is non null }
\end{array}
\end{array}\right.
$$

where $\mathbf{x}_{3}=\varphi_{2}\left(\varphi_{1}\left(R\left(\mathbf{e}_{3}\right)\right)\right)-\mathbf{e}_{3}$. By the very construction of $\varphi_{3}$, we have $\varphi_{3}\left(\varphi_{2}\left(\varphi_{1}\left(R\left(\mathbf{e}_{3}\right)\right)\right)\right)=\mathbf{e}_{3}$. Since $\varphi_{2} \circ \varphi_{1} \circ R$ is an orthogonal transformation, $\varphi_{2}\left(\varphi_{1}\left(R\left(\mathbf{e}_{1}\right)\right)\right)$ and is $\varphi_{2}\left(\varphi_{1}\left(R\left(\mathbf{e}_{2}\right)\right)\right)$ are orthogonal to $\varphi_{2}\left(\varphi_{1}\left(R\left(\mathbf{e}_{3}\right)\right)\right)$. Hence $\mathbf{e}_{1}, \mathbf{e}_{2} \in \mathbf{x}_{3}^{\perp}$ then $\varphi_{3}\left(\mathbf{e}_{1}\right)=\mathbf{e}_{1}$ and $\varphi_{3}\left(\mathbf{e}_{2}\right)=\mathbf{e}_{2}$. Therefore, we have

$$
\varphi_{3}\left(\varphi_{2}\left(\varphi_{1}\left(R\left(\mathbf{e}_{i}\right)\right)\right)\right)=\mathbf{e}_{i}, \quad i=1,2,3 .
$$

Thus we have also

$$
\varphi_{3} \circ \varphi_{2} \circ \varphi_{1} \circ R=I_{3} .
$$

Consequently, the orthogonal transformation $R$ can be expressed as the following product of three simple Lorentzian reflections

$$
R=\varphi_{1}^{-1} \circ \varphi_{2}^{-1} \circ \varphi_{3}^{-1}=\varphi_{1} \circ \varphi_{2} \circ \varphi_{3} .
$$

Remark 10. If the number of reflections that decompose a given orthogonal transformation is even (odd) then the transformation is a Lorentzian rotation (reflection).

\section{Applications}

Example 11. Let us find the Lorentzian reflection matrix with the plane of reflection

$$
\mathbf{x}^{\perp}=\left\{\left(y_{1}, y_{2}, y_{3}\right) \in \mathbb{E}_{1}^{3} ; y_{1}-y_{2}+2 y_{3}=0\right\} .
$$

Here $\mathbf{x}=(-1,-1,2)$. By using Theorem 2, we find

$$
\varphi_{\mathbf{x}}=\left[\begin{array}{ccc}
3 / 2 & -1 / 2 & 1 \\
1 / 2 & 1 / 2 & 1 \\
-1 & 1 & -1
\end{array}\right]
$$


Example 12. Let us find the plane of reflection for the Lorentzian reflection matrix

$$
\varphi=\left[\begin{array}{ccr}
3 / 2 & -1 & -1 / 2 \\
1 & -1 & -1 \\
1 / 2 & -1 & 1 / 2
\end{array}\right] .
$$

By long and tedious calculations, we find the eigenvector of $\varphi$ corresponding to the eigenvalue -1 as $\mathbf{x}=(1,2,1)$. So the plane of reflection is found as

$$
\mathbf{x}^{\perp}=\left\{\left(y_{1}, y_{2}, y_{3}\right) \in \mathbb{E}_{1}^{3} ;-y_{1}+2 y_{2}+y_{3}=0\right\} .
$$

Example 13. Consider the spacelike vectors $\mathbf{u}=(-1,1,-1)$ and $\mathbf{v}=(7,5,-5)$ such that $\mathbf{u} \neq \mathbf{v}$ and $\varepsilon_{\mathbf{u}}=\varepsilon_{\mathbf{v}}=1$. By Theorem 8 there exists a unique Lorentzian reflection $R: \mathbb{E}_{1}^{3} \rightarrow \mathbb{E}_{1}^{3}$ such that $R(\mathbf{u})=\mathbf{v}$. Since if $\mathbf{v} \neq-\mathbf{u}$ and $\mathbf{u}-\mathbf{v}=$ $(-8,-4,4)$ is non null, then the Lorentzian rotation $R$ is defined as

$$
R(\mathbf{x})=-\varphi_{\mathbf{v}}\left(\varphi_{\mathbf{u}-\mathbf{v}}(\mathbf{x})\right) .
$$

By long and tedious calculations, we find the eigenvalues of the rotation matrix as follows

$$
R(\mathbf{x})=\left(-17 x_{1}-12 x_{2}+12 x_{3},-12 x_{1}-8 x_{2}+9 x_{3}, 12 x_{1}+9 x_{2}-8 x_{3}\right) .
$$

The matrix representation of $R$ is

$$
R_{\left\{e_{1}, e_{2}, e_{3}\right\}}=\left[\begin{array}{rrr}
-17 & -12 & 12 \\
-12 & -8 & 9 \\
12 & 9 & -8
\end{array}\right] .
$$

It is easily seen that $R(\mathbf{u})=\mathbf{v}$.

Example 14. Consider the following orthogonal transformation

$$
R\left(x_{1}, x_{2}, x_{3}\right)=\left(\frac{9}{4} x_{1}-2 x_{2}+\frac{1}{4} x_{3}, x_{2}-x_{1}-x_{3}, 2 x_{2}-\frac{7}{4} x_{1}+\frac{1}{4} x_{3}\right)
$$

which has matrix representation as

$$
R_{\left\{\mathbf{e}_{1}, \mathbf{e}_{2}, \mathbf{e}_{3}\right\}}=\left[\begin{array}{ccc}
9 / 4 & -2 & 1 / 4 \\
-1 & 1 & -1 \\
-7 / 4 & 2 & 1 / 4
\end{array}\right] .
$$

Here $\left\{\mathbf{e}_{1}, \mathbf{e}_{2}, \mathbf{e}_{3}\right\}$ is the standard basis for $\mathbb{E}_{1}^{3}$.

Step 1: First consider $\mathbf{v}=\mathbf{e}_{1}=(1,0,0)$ and $\mathbf{u}=R\left(\mathbf{e}_{1}\right)=(9 / 4,-1,-7 / 4)$. Since $R\left(\mathbf{e}_{1}\right) \neq \mathbf{e}_{1}$, then there exists a simple Lorentzian reflection $\varphi_{1}$ such that 
$\varphi_{1}\left(R\left(\mathbf{e}_{1}\right)\right)=\mathbf{e}_{1}$ according to Theorem 2 . And $R\left(\mathbf{e}_{1}\right) \neq-\mathbf{e}_{1}$ and $R\left(\mathbf{e}_{1}\right)-\mathbf{e}_{1}=$ $(5 / 4,-1,-7 / 4)$ is non null. By Theorem 10, the simple Lorentzian reflection $\varphi_{1}$ is defined as well because there exists a simple reflection

$$
\begin{aligned}
\varphi_{1}(\mathbf{x}) & =\varphi_{R\left(\mathbf{e}_{1}\right)-\mathbf{e}_{1}}(\mathbf{x})=\varphi_{(5 / 4,-1,-7 / 4)}(\mathbf{x}) \\
& =\left(\frac{9}{4} x_{1}+x_{2}+\frac{7}{4} x_{3},-x_{1}+\frac{1}{5} x_{2}-\frac{7}{5} x_{3},-\frac{7}{4} x_{1}-\frac{7}{5} x_{2}-\frac{29}{20} x_{3}\right)
\end{aligned}
$$

with matrix representation

$$
\varphi_{1\left\{\mathbf{e}_{1}, \mathbf{e}_{2}, \mathbf{e}_{3}\right\}}=\left[\begin{array}{ccc}
9 / 4 & 1 & 7 / 4 \\
-1 & 1 / 5 & -7 / 5 \\
-7 / 4 & -7 / 5 & -29 / 20
\end{array}\right]
$$

Here $\varphi_{1}\left(R\left(\mathbf{e}_{2}\right)\right)=(0,-3 / 5,-4 / 5)$ and $\varphi_{1}\left(R\left(\mathbf{e}_{3}\right)\right)=(0,-4 / 5,3 / 5)$. That is

$$
\varphi_{1}\left(R\left(\mathbf{e}_{i}\right)\right) \in \operatorname{Span}\left\{\mathbf{e}_{2}, \mathbf{e}_{3}\right\}, \quad i=2,3 .
$$

Step 2: Now consider $\mathbf{v}=\mathbf{e}_{2}=(0,1,0)$ and $\mathbf{u}=\varphi_{1}\left(R\left(\mathbf{e}_{2}\right)\right)=(0,-3 / 5,-4 / 5)$. Since $\varphi_{1}\left(R\left(\mathbf{e}_{2}\right)\right) \neq \mathbf{e}_{2}$ then there exists a simple Lorentzian reflection $\varphi_{2}$ such that $\varphi_{2}\left(\varphi_{1}\left(R\left(\mathbf{e}_{2}\right)\right)\right)=\mathbf{e}_{2}$ according to Theorem 2 . We know that $\varphi_{1}\left(R\left(\mathbf{e}_{2}\right)\right) \neq-\mathbf{e}_{2}$ and $\varphi_{1}\left(R\left(\mathbf{e}_{2}\right)\right)-\mathbf{e}_{2}=(0,-8 / 5,-4 / 5)$ is non null. Application of Theorem 8 yields that the simple reflection $\varphi_{2}$ is defined as follows

$\varphi_{2}(\mathbf{x})=\varphi_{\varphi_{1}\left(R\left(\mathbf{e}_{2}\right)\right)-\mathbf{e}_{2}}(\mathbf{x})=\varphi_{(0,-8 / 5,-4 / 5)}(\mathbf{x})=\left(x_{1},-\frac{3}{5} x_{2}-\frac{4}{5} x_{3},-\frac{4}{5} x_{2}+\frac{3}{5} x_{3}\right)$.

Here the matrix representation of the Lorentzian reflection is obtained as

$$
\varphi_{2\left\{\mathbf{e}_{1}, \mathbf{e}_{2}, \mathbf{e}_{3}\right\}}=\left[\begin{array}{ccc}
1 & 0 & 0 \\
0 & -3 / 5 & -4 / 5 \\
0 & -4 / 5 & 3 / 5
\end{array}\right]
$$

Moreover, we get

$$
\varphi_{2}\left(\varphi_{1}\left(R\left(\mathbf{e}_{i}\right)\right)\right)=\mathbf{e}_{i}, \quad i=1,2,3 .
$$

Thus, the orthogonal transformation $R$ can be expressed as decomposition of two simple Lorentzian reflections

$$
R=\varphi_{1} \circ \varphi_{2}
$$

that is

$$
R=\left[\begin{array}{ccl}
9 / 4 & -2 & 1 / 4 \\
-1 & 1 & -1 \\
-7 / 4 & 2 & 1 / 4
\end{array}\right]=\left[\begin{array}{ccc}
9 / 4 & 1 & 7 / 4 \\
-1 & 1 / 5 & -7 / 5 \\
-7 / 4 & -7 / 5 & -29 / 20
\end{array}\right]\left[\begin{array}{ccc}
1 & 0 & 0 \\
0 & -3 / 5 & -4 / 5 \\
0 & -4 / 5 & 3 / 5
\end{array}\right]
$$

Consequently, $R$ is a Lorentzian rotation. 


\section{References}

[1] Brezov D., Mladenova C. and Mladenov I., The Geometry of Phytagorean Quadruples and Rational Decomposition of Pseudo-Rotations, In: Algorithms in Industirial Mathematics, A. Spasova (Ed), Cambridge Scholars Publishing, Cambridge 2015, pp 210-234.

[2] Cockle J., On Systems of Algebra Involving More than One Imaginary, Phil. Mag. 35 (1849) 434-435.

[3] Gonzalez G., Aragon J., Rodriguez-Andrade M. and Verde-Star L., Reflections, Rotations and Pythagorean Numbers, Adv. Appl. Clifford Algebras 19 (2009) 1-14.

[4] Gonzalez G., Aragon J. and Rodriguez-Andrade M., Pythagorean Vectors and Clifford Numbers, Adv. Appl. Clifford Algebras 21 (2011) 247-258.

[5] Naber G., The Geometry of Minkowski Spacetime: An Introduction to the Mathematics of the Special Theory of Relativity, Springer, New York 2012.

[6] Özdemir M. and Ergin A., Rotations with Unit Timelike Quaternions in Minkowski 3-Space, J. Geom. Phys. 56 (2006) 322-336.

[7] Özdemir M., The Roots of a Split Quaternion, Appl. Math. Lett. 22 (2009) 258-263.

[8] Özdemir M., Erdoğdu M. and Şimşek H., On the Eigenvalues and Eigenvectors of a Lorentzian Rotation Matrix by Using Split Quaternions, Adv. Appl. Clifford Algebras 24 (2014) 179-192.

[9] Rodriguez-Andrade M., Gonzalez G., Aragon J. and Verde-Star L., An Algorithm for Cartan-Dieudonne Theorem on Generalized Scalar Product Space, Lin. Alg. Appl. 434 (2011) 1238-1254.

Received 08 July 2015

Melek Erdoğdu

Department Math \& Computer Sciences

Necmettin Erbakan University

Konya 42090, TURKEY

E-mail address: merdogdu@konya.edu.tr

Mustafa Özdemir

Department of Mathematics

Akdeniz University

Antalya, 07058, TURKEY

E-mail address: mozdemir@akdeniz.edu.tr 